\title{
1-Trichloromethyl-1,2,3,4-tetrahydro-beta-carboline (TaClo) Induces the Apoptosis of Dopaminergic Neurons via Oxidative Stress and Neuroinflammation
}

\author{
Yihang Yang $\left(D,{ }^{1}\right.$ Bo Pang $\left(D,{ }^{2}\right.$ Zihao Liu, ${ }^{3}$ Jie Li, ${ }^{1}$ Zhen Zhang, ${ }^{1}$ Rui Zhang, ${ }^{1}$ Xianzeng Hou, ${ }^{4}$ \\ Hua Guo, ${ }^{1}$ Lingyi Chi, ${ }^{5}$ Qi Pang, ${ }^{1}$ and Tao Xin $\mathbb{1}^{1,6}$ \\ ${ }^{1}$ Department of Neurosurgery, Shandong Provincial Hospital Affiliated to Shandong University, Jinan, Shandong 250021, China \\ ${ }^{2}$ Department of Neurosurgery, Qilu Hospital of Shandong University, Jinan, Shandong 250012, China \\ ${ }^{3}$ Medical College of Nanchang University, Nanchang, Jiangxi 330000, China \\ ${ }^{4}$ Department of Neurosurgery, Qianfoshan Hospital affiliated to Shandong University, Shandong 250014, China \\ ${ }^{5}$ Department of Neurosurgery, Qilu Hospital of Shandong University and Brain Science Research Institute of Shandong University, \\ Jinan, 250012 Shandong, China \\ ${ }^{6}$ Department of Neurosurgery, Jiangxi Provincial People's Hospital Affiliated to Nanchang University, Nanchang 330006, \\ Jiangxi, China
}

Correspondence should be addressed to Tao Xin; docxintao@163.com

Received 19 November 2018; Accepted 21 January 2019; Published 7 March 2019

Academic Editor: Ryuichi Morishita

Copyright (C) 2019 Yihang Yang et al. This is an open access article distributed under the Creative Commons Attribution License, which permits unrestricted use, distribution, and reproduction in any medium, provided the original work is properly cited.

\begin{abstract}
Several in vitro studies have revealed the neurotoxicity of 1-trichloromethyl-1,2,3,4-tetrahydro-beta-carboline (TaClo). However, the underlying mechanism has not been completely elucidated, particularly in vivo. This study was designed to study the neurotoxicity of TaClo in vivo by stereotactically injecting TaClo into the striatum of Wistar rats. After the TaClo injections, rats were subjected to an open field test, and their distance travelled and tracks showed decreasing trends over time. The results of liquid chromatography-mass spectrometry analysis showed that the motor dysfunction of the TaClo-treated rats was accompanied by reduced dopamine levels in the striatum. Based on the diffusion tensor imaging data, the apparent diffusion coefficient of the nigrostriatal pathway was significantly increased, and subsequent histological staining revealed the demyelination of nigrostriatal fibres after the TaClo treatment. TaClo induced a loss of tyrosine hydroxylase-positive cells in the substantia nigra compacta. Regarding the underlying mechanism, TaClo caused oxidative stress in the nigrostriatal system by increasing the production of reactive oxygen species and reducing the mitochondria membrane potential. Meanwhile, the elevated expression of Iba-1, TNF- $\alpha$, IL-6, Cox-2, and iNOS indicated microglial activation and a strong innate immune response in the nigrostriatal system. In addition, activated caspase-3 levels were increased. Thus, both mitochondrial impairments and the innate immune response are involved in TaClo-induced neurotoxicity.
\end{abstract}

\section{Introduction}

Parkinson's disease (PD) is one of the most common neurodegenerative movement disorders and affects approximately $1-2 \%$ of elderly people. Both genetic and environmental factors are strongly correlated with the development of PD. However, to date, the exact aetiology and underlying molecular mechanisms of PD remain largely unclear [1]. 1-Methy-1-4-phenyl-1,2,3,6-tetrahydropyridine (MPTP) is known to cause PD. Many other structural analogues of MPTP have been discovered in the environment, including herbicides (e.g., paraquat), alkaloids (e.g., 1,2,3,4-tetrahydroisoquinolines), and $\beta$-carbolines [2]. Trichloroethylene (TCE) is widely used as a detergent, extractant, and solvent. The main routes of TCE exposure are industrial waste gas, contaminated groundwater, and volatile organic solvents. 1-Trichloromethyl-1,2,3,4-tetrahydro-beta-carboline (TaClo) is an in vivo metabolic product of TCE. TaClo belongs to 
the $\beta$-carboline family and has a structure similar to the synthetic neurotoxin 1-methyl-4-phenylpyridinium iodide $\left(\mathrm{MPP}^{+}\right)$[3]. Previous studies have reported TaClo concentrations ranging from less than $1 \mathrm{ng}$ to $35 \mathrm{ng}$ per millilitre in blood samples from patients who were orally treated with chloral hydrate for 3 days to 6 months [4, 5]. Moreover, TaClo is approximately 10 times more potent than MPTP [6], and it penetrates the blood-brain barrier more easily than MPTP [7]. However, to date, the mechanism by which TaClo induces $\mathrm{PD}$ remains unclear.

The nigrostriatal pathway (NP) is an anatomical circuit comprising dopaminergic neurons that project from the substantia nigra compacta $(\mathrm{SNc})$ to the striatum forming part of the basal ganglia motor loop. Dopaminergic neurons in the SNc and their terminals in the striatum constitute the nigrostriatal dopaminergic system. This system is easily impaired because many extracellular materials can be taken up via plasma membrane dopamine transporters [8]. We hypothesized that TaClo causes PD symptoms by damaging the nigrostriatal system. Mitochondrial dysfunction, oxidative stress, and inflammation are involved in degenerative diseases [9]. As shown in the study by Prof. Bing G., TCE treatment impairs mitochondrial complex I activity [10]. Furthermore, by referring to the GEO database (GSE7621-GPL570), which provides analyses of the SNc in postmortem brain tissues from patients, the expression of the Cox-2, iNOS, GABPA, and Keap 1 genes is upregulated in patients with $\mathrm{PD}$. We also hypothesized that TaClo induces a positive feedback loop between mitochondrial oxidative stress and the innate immune response in the central nervous system (CNS). Mitochondrial inhibitors, such as rotenone and MPTP, are known to increase reactive oxygen species (ROS) production by blocking the electron transport chain at a particular site [11], further causing abnormalities in neuroglia [12]. More importantly, substantially elevated ROS levels are implicated in the activation of the innate immune response [13]. Subsequently, activated microglia release ROS, further impairing the mitochondria. This type of positive feedback between mitochondrial dysfunction and the inflammatory cascade in the brain may cause secondary injury, such as demyelination, dopaminergic neuron apoptosis, and neurodegenerative diseases.

\section{Materials and Methods}

2.1. Experimental Animals. Fifty-five male Wistar rats aged between 8 and 10 weeks (weight 260-300 g, purchased from Vital River Laboratory Animal Technology Co. Ltd., Beijing, China) were included in this experimental study. All animals were housed under standard conditions with a $12 \mathrm{~h}$ day/night cycle and free access to food and water (room temperature $22^{\circ} \mathrm{C}$, humidity 55\%). The experimental animals were randomly divided into two groups: a control group $(n=25$; $272.3 \pm 9.6 \mathrm{~g})$ and a TaClo group $(n=25 ; 276.9 \pm 7.8 \mathrm{~g})$. Rats in the TaClo group were stereotactically injected with TaClo (provided by the laboratory of Dr. Bing Guoying, USA) dissolved in polyethylene glycol (PEG) (P3015, Sigma-Aldrich, St Louis, MO, USA) into the right striatum. The control rats were injected with PEG. All procedures were performed in accordance with the guidelines and regulations of the Experimental Centre of Shandong Provincial Hospital and approved by the Ethics Committee of Shandong Provincial Hospital affiliated to Shandong University.

2.2. Stereotactic Injection. Rats $(n=25 ; 276.9 \pm 7.8 \mathrm{~g})$ were anaesthetized with an intraperitoneal injection of ketamine. After being anaesthetized, the animals were placed in a stereotaxic apparatus ( $\mathrm{ZH}-\mathrm{B}$, Zhenghua Biological Instrument Equipment Co., Ltd, China). The stereotactic injections were performed with a $1 \mu \mathrm{l}$ microsyringe (Hamilton, Sweden). The following stereotactic coordinates were used for the unilateral intrastriatal injections relative to the bregma: site 1: anteroposterior (AP) $1.0 \mathrm{~mm}$, mediolateral (ML) $2.0 \mathrm{~mm}$, and dorsoventral (DV) $5.5 \mathrm{~mm}$; site $2:$ AP $1.0 \mathrm{~mm}$, $\mathrm{ML} 3.5 \mathrm{~mm}$, and DV $6.0 \mathrm{~mm}$; site $3: \mathrm{AP}-0.5 \mathrm{~mm}, \mathrm{ML}$ $2.5 \mathrm{~mm}$ and DV $5.0 \mathrm{~mm}$; and Site $4: \mathrm{AP}-0.5 \mathrm{~mm}, \mathrm{ML}$ $4.0 \mathrm{~mm}$ and DV $6.5 \mathrm{~mm}$. TaClo was dissolved in PEG at a concentration of $2.50 \mu \mathrm{g} / \mu \mathrm{l}$. Considering that rats weighing $250 \mathrm{~g}$ to $350 \mathrm{~g}$ have similar brain volumes, each rat was injected with the same amount of TaClo corresponding to $1 \mu \mathrm{l}$ per injection site. The total dose was $10 \mu \mathrm{g}$ of TaClo per rat [14]. The injection rate was $0.25 \mu \mathrm{l} / \mathrm{min}$, and the syringe remained in the injection site for an additional $5 \mathrm{~min}$ before being retracted. After the injection, each rat was monitored until it recovered from the anaesthesia. The control animals ( $n=25 ; 272.3 \pm 9.6 \mathrm{~g})$ were stereotactically injected with PEG. Rats in the sham group $(n=3 ; 269.5 \pm 6.3 \mathrm{~g})$ received only surgery; they did not receive any injections and served as a control to exclude possible effects of the surgical procedures on the results of the diffusion tensor imaging (DTI) test.

2.3. Open Field Test. Rats $(n=5)$ were recorded with an automatic video tracking system (ZH-ZFT, Zhenghua Biological Instrument Equipment Co. Ltd, China). Rats in both the TaClo group and the control group were placed in the testing box $(40 \times 40 \times 35 \mathrm{~cm})$ in a random order. Before the test, each rat was allowed to adapt to its surroundings for $10 \mathrm{~min}$. Then, during the following $30 \mathrm{~min}$, the movements of the rats, including the tracks and total distance travelled, were recorded by the video tracking system.

2.4. DTI. Rats $(n=3)$ were anaesthetized before the test. The test was performed by an imaging technician. Conventional MRI and DTI scans were conducted on a 3.0 T MRI scanner (Philips Achieva TX, Best, The Netherlands) with a wrist coil. Rats were placed in the scanner in the prone position. Conventional MRI scans, including T1-weighted and T2-weighted sagittal images, were completed with the SE sequence. DTI images were acquired using a spin-echo planar imaging sequence. After acquiring the images, the data were transferred to a Philips workstation to calculate the DTI data. For accurate positioning, the DTI images were mixed with sagittal T2-weighted images and the regions of interest (ROIs) in the striatum and the SNc were defined. The parameters, including the apparent diffusion coefficient (ADC) and fractional anisotropy (FA) values, were analysed. The averages of multiple measurements were considered the final results. 
2.5. Luxol Fast Blue (LFB) Staining. Rats were euthanatized with carbon dioxide. The brains were removed, fixed with $4 \%$ paraformaldehyde for $48 \mathrm{~h}$, and then embedded in paraffin. The sections were cut at a thickness of $10 \mu \mathrm{m}$ using a microtome and stained with LFB to reveal the demyelinated areas according to the manufacturer's protocol (American MasterTech, CA, USA).

2.6. Liquid Chromatography-Tandem Mass Chromatography (LC-MS/MS) Analysis. Rat striatal tissues weighing 50-60 mg were homogenized in a solution containing $200 \mu \mathrm{l}$ of ultrapure water $(0.1 \%$ formic acid) and $800 \mu \mathrm{l}$ of ice-cold methanol/acetonitrile $(1: 1, v / v)$. The homogenate was vortex-mixed for $1 \mathrm{~min}$ and ultrasonically lysed on ice for $30 \mathrm{~min}$. Then, the homogenate was centrifuged at $14,000 \times \mathrm{g}$ for $20 \mathrm{~min}$ at $4^{\circ} \mathrm{C}$. The supernatant was transferred to a new tube and evaporated to dryness under a nitrogen stream. The dry residue was reconstituted in $100 \mu \mathrm{l}$ of the initial mobile phase $(0.1 \%$ formic acidin water/acetonitrile, $1: 1, v /$ $v$ ) and centrifuged at $14,000 \times g$ for $15 \mathrm{~min}$. Then, the supernatant was injected into the LC-MS system for analysis. The MS-mediated acquisition of dopamine (DA), $\gamma$-amino-butyric acid (GABA), epinephrine (Epi), glutamine, glutamate, 5-hydroxyindole-3-acetic acid (5-HIAA), and serotonin (5-HT) spectra was performed in the electrospray positive ionization multiple reaction monitoring (MRM) mode.

2.7. Immunofluorescence (IF) Staining. For the IF analysis, frozen sections were permeabilized with $0.2 \%$ Triton X-100 for $20 \mathrm{~min}$ and blocked with goat serum for $1 \mathrm{~h}$. Then, the slides were incubated with a rabbit monoclonal anti-tyrosine hydroxylase $(\mathrm{TH})$ primary antibody $(1: 200$, ab75875, Abcam, USA) at $4^{\circ} \mathrm{C}$ overnight. The slides were washed with PBS 3 times for $5 \mathrm{~min}$ each and incubated with an Alexa 488-conjugated goat-anti-rabbit secondary antibody (1:200, ab150077, Abcam, USA). The excitation wavelength used for the assay is $490 \mathrm{~nm}$, and the emission wavelength is $520 \mathrm{~nm}$.

2.8. Nissl Staining. Paraffin sections were routinely dewaxed and incubated with toluidine blue in a $55^{\circ} \mathrm{C}$ incubator for $40 \mathrm{~min}$. Sections were washed with distilled water 3 times for $5 \mathrm{~min}$ each. After differentiation with hydrochloric acid in alcohol, sections were dehydrated and sealed with neutral gum.

2.9. Flow Cytometry (FCM) Analysis. For staining, the tissue was minced and digested with trypsin for $15 \mathrm{~min}$ at $37^{\circ} \mathrm{C}$. Then, the tissue homogenate was filtered through a $40 \mu \mathrm{m}$ filter and prepared using a fixation/permeabilization solution according to the manufacturer's instructions (BD Pharmingen, San Diego, CA). Cells were incubated with a primary rabbit-anti-rat $\mathrm{TH}(1: 200, \mathrm{ab75875}$, Abcam $)$ at $4^{\circ} \mathrm{C}$ for $40 \mathrm{~min}$. After washes with PBS, cells were incubated with a goat-anti-rabbit-PE secondary antibody ( $1: 100$, ab7010, Abcam, USA). The cells were washed with PBS again. Then, cells were stained with a ROS dye (Invitrogen, Carlsbad, USA) at $37^{\circ} \mathrm{C}$ for $1 \mathrm{~h}$ according to the manufacturer's protocol. After washes with PBS, multiple colour fluorescence-activated cell sorting (FACS) analyses
TABLE 1

\begin{tabular}{|c|c|}
\hline \multirow{2}{*}{ GADPH } & Forward $5^{\prime}$-AACCTGCCAAGTATGATGAC-3' \\
\hline & Reverse 5'-GCTGTAGCCATATTCATTGT-3' \\
\hline \multirow{2}{*}{ IL-6 } & Forward 5'-CTGGAGTTCCGTTTCTACCT-3' \\
\hline & Reverse 5'-GCCACTCCTTCTGTGACTCT-3' \\
\hline \multirow{2}{*}{ TNF- $\alpha$} & Forward 5'-GCCAATGGCATGGATCTC-3' \\
\hline & Reverse $5^{\prime}$-TGTCCTTAGGGCAAGGGCT-3' \\
\hline \multirow{2}{*}{$\operatorname{Cox} 2$} & Forward 5'-CTTCGGAATTCAATCATGAG-3' \\
\hline & Reverse $5^{\prime}$-TCCAGAACTTCTTTTGAATC-3' \\
\hline \multirow{2}{*}{ iNOS } & Forward 5 '-ACATCTGGCAGGATGAGAAG-3' \\
\hline & Reverse 5'-CTTGTTGTTGAAGGCGTAGC-3' \\
\hline
\end{tabular}

were performed by using a FACSCalibur cytometer (Beckman Coulter, KBB, CA, USA). The data were analysed using FlowJo software (TreeStar, San Carlos, CA). First, the TH-positive cells were sorted. The ROS mean fluorescence intensity in these TH-positive cells was measured and analysed.

2.10. JC-1 Staining. The brains were immediately embedded in O.C.T (Tissue-Tek, Sakura, USA). In addition, $10 \mu \mathrm{m}$ sections were cut using a freezing microtome. Changes in the mitochondrial membrane potential were measured by incubating the tissues with $5,5^{\prime} 6,6^{\prime}$-tetrachloro- $1,1^{\prime} 3,3^{\prime}$-tetraethylbenzimidazole-carbocyanine iodide (JC-1, Beyotime Biotechnology, China) for $20 \mathrm{~min}$ at $37^{\circ} \mathrm{C}$. Aggregates of the fluorescent JC-1 probe yield red fluorescence with an emission wavelength of $590 \mathrm{~nm}$. In addition, the monomer form of the fluorescent JC-1 probe yields green fluorescence with an emission wavelength of $530 \mathrm{~nm}$.

2.11. ATP Measurement. ATP levels were measured using a luciferin-luciferase-based ATP assay kit (Beyotime, China). The working solution was prepared according to the manufacturer's protocol. One hundred microliters of working solution was added to each well of a 96-well plate. The 96-well plate was incubated at room temperature for $5 \mathrm{~min}$. Then, the ATP standards and samples were added. Luciferase activity was detected with a microplate reader (Synergy H1, BioTek, USA). The ATP level was calculated according to the standard curve, normalized to the protein contents in each sample and reported as nmol/mg of protein.

2.12. Immunohistochemistry (IHC). Rats $(n=3)$ were euthanized and perfused with $150 \mathrm{ml}$ of saline and $300 \mathrm{ml}$ of $4 \%$ paraformaldehyde. Brain tissues were collected and fixed with $4 \%$ paraformaldehyde for $48 \mathrm{~h}$. Using a rat brain mould, brain tissues were cut at the same coronal plane of the SNc. Then, $10 \mu \mathrm{m}$-thick coronal slices were prepared. Antigens were retrieved in citric acid buffer (pH6.0) using a high-pressure antigen retrieval method. Every sixth coronal section from the region was incubated with rabbit anti-Iba-1 (1:500, 019-19741, Wako, Japan) and rabbit monoclonal anti- $\alpha$-synuclein primary antibodies $(1: 200$, 


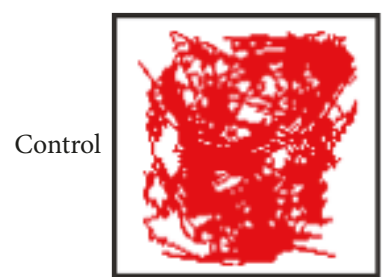

$7 \mathrm{~d}$

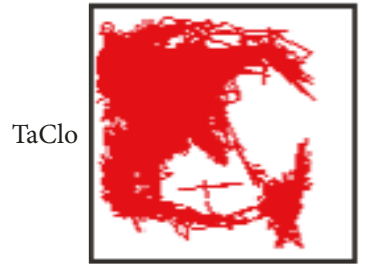

$7 \mathrm{~d}$

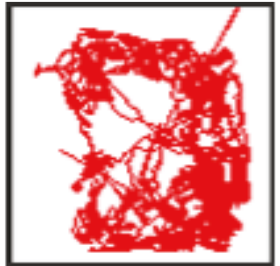

$14 \mathrm{~d}$

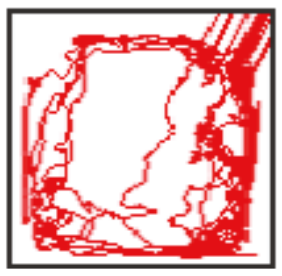

$14 \mathrm{~d}$

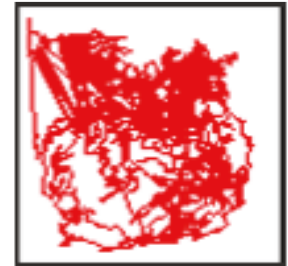

$21 \mathrm{~d}$

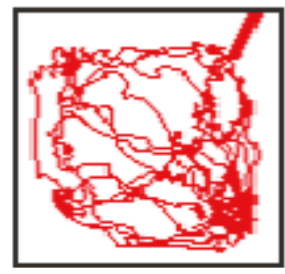

$21 \mathrm{~d}$

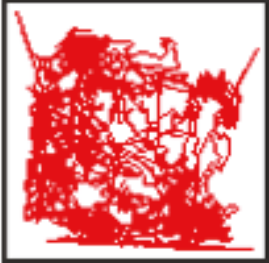

$28 \mathrm{~d}$

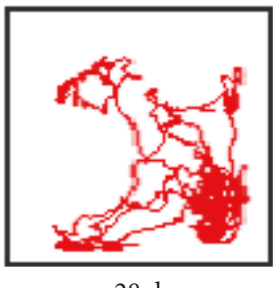

$28 \mathrm{~d}$

(a)

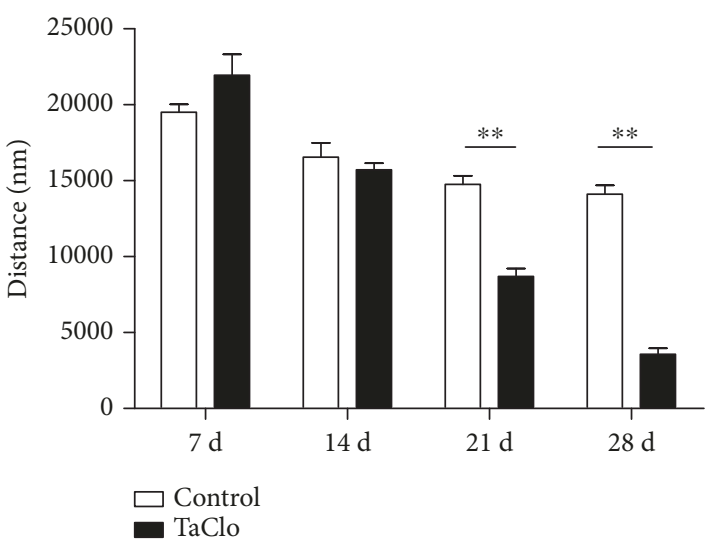

(b)

Figure 1: TaClo induced motor ability impairments. ( $a$ and $b$ ) The open field test revealed that symptoms of bradykinesia in the TaClo-injected rats on the 21 st and 28 th days after injection compared to the control rats. ${ }^{* *} p<0.01$ compared to the control group.

ab51253, Abcam, USA) at $4^{\circ} \mathrm{C}$. Then, the immunoreactive cells were visualized using streptavidin-peroxidase-conjugated goat-anti-rabbit secondary antibody (SP kits, PV-9000, ZSGB-Bio Co. Ltd., Beijing, China). In addition, the mean integrated optical density (IOD) of the positive staining was measured using Image-Pro Plus software (Media Cybernetics, USA). Six equal areas from each section were randomly selected to measure the area of positive staining. Five sections from each sample were counted, and the average value was calculated.

2.13. Quantitative Real-Time PCR. Total RNA was isolated using TRIzol reagent (Invitrogen). Alterations in the mRNA levels of selected genes were measured using RT-PCR following the reverse transcription of $1 \mu \mathrm{g}$ of RNA from each sample using a PrimeScript RT reagent kit (Takara Bio Inc., Otsu, Japan). Quantitative RT-PCR was performed on a LightCycler instrument (Roche Diagnostics, Mannheim, Germany) with SYBR Premix Ex Taq technology (Takara Bio Inc., Otsu, Japan) according to the manufacturer's protocol. The relative mRNA levels were determined using the $2^{-\Delta \mathrm{Ct}}$ method. The primer sequences used to determine the expression of specific genes are listed in Table 1.
2.14. Western Blot Analysis. An equal amount of proteins $(40 \mu \mathrm{g})$ was separated using SDS-polyacrylamide gel electrophoresis and transferred to polyvinylidene fluoride membranes. Membranes were blocked with 5\% skim milk and incubated with primary antibodies against TH (monoclonal, 1:10000, ab75875, Abcam, USA), TNF- $\alpha$ (monoclonal 1:1000, 17590-1-AP, Proteintech, USA), IL-6 (monoclonal, 1:1000, sc-57315, Santa Cruz, USA), Cox-2 (monoclonal, $1: 1000,12282$, CST, USA), iNOS (polyclonal, 1:500, 18985-1-AP, Proteintech, USA), and caspase-3 (polyclonal $1: 1000,19677-1-A P$, Proteintech, USA) overnight at $4^{\circ} \mathrm{C}$. Membranes were then incubated with the appropriate secondary antibodies (anti-rabbit IgG, ZB-2301, and anti-rat IgG, ZB-2305, ZSGB-BIO, China) at room temperature for one hour and washed with TBST three times. Membranes were visualized using a Digital Imaging System and analysed using AlphaView software.

2.15. Equipment and Settings. The western blotting results were obtained using a luminescent image analyser (Amersham Imager 600, Sweden). An optical microscope (Leica DM4000 B, Germany) was used to capture the images of IHC staining. A fluorescence microscope (Carl Zeiss A2, 


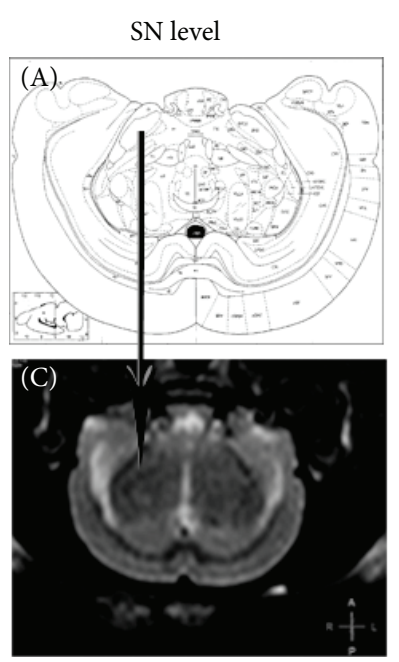

(a)

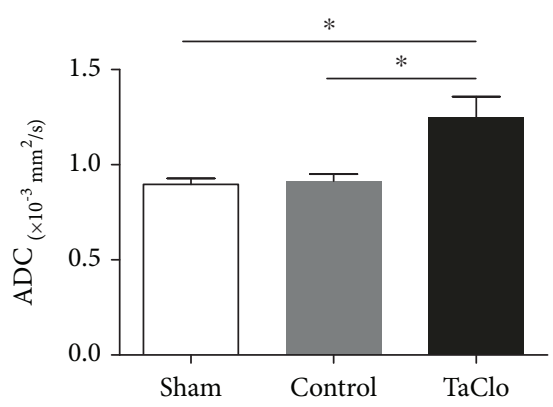

(c)

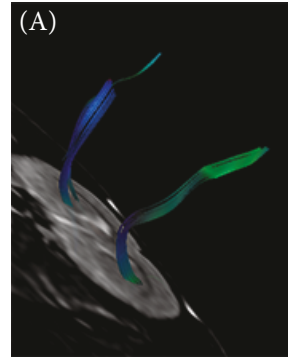

Sham group

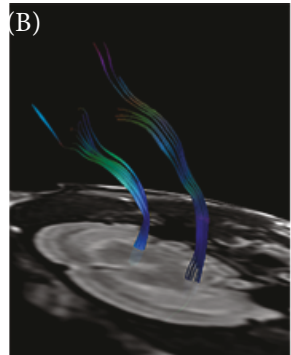

Control group

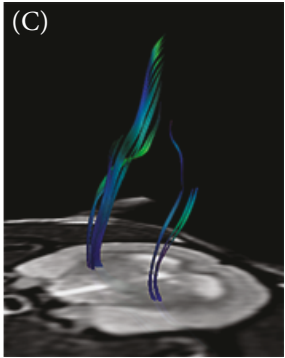

TaClo group

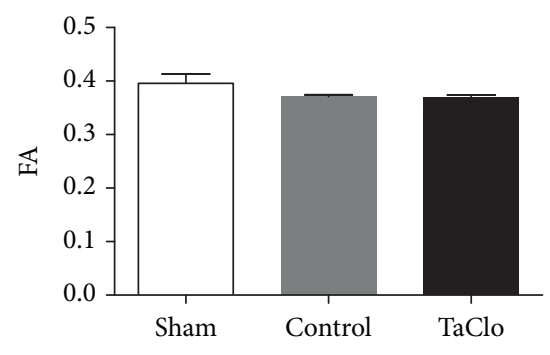

(d)

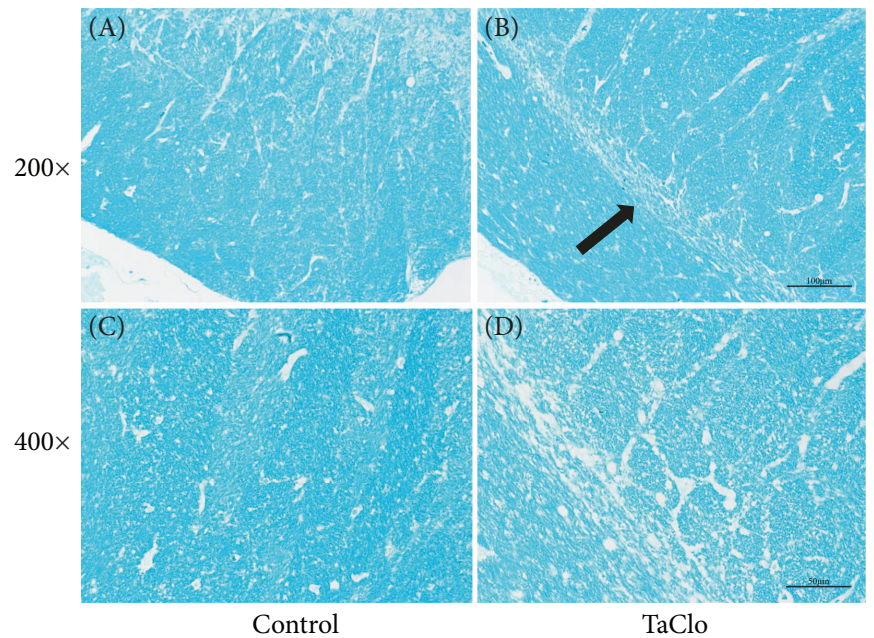

(e)

FIgure 2: TaClo impaired the nigrostriatal system. (a) Comparison of stereotaxic coordinates in an atlas of the rat brain and the ROI assessed using DTI. Arrows pointing from A to C and from B to C show the SNc and striatum, respectively. (b) Images of the general outline of the nigrostriatal system indicate the obvious destruction of this region in the TaClo-injected rats compared to the other two groups. (c) Histogram showing a significant increase in the ADC value for the nigrostriatal system after TaClo injection. ${ }^{*} p<0.05$ compared to the control group. ${ }^{*} p<0.05$ compared to the sham group. (d) The FA value for the NP did not significantly change. (e) LFB staining showing demyelination in the SNc of the TaClo-treated rats (arrow). 
TABLE 2: Neurotransmitter levels in the rat striatum (mean \pm SEM).

\begin{tabular}{lcc}
\hline & Control & TaClo \\
\hline Dopamine (ng/g) & $11.267 \pm 1.079$ & $4.645 \pm 1.097^{* *}$ \\
Glutamine (ng/g) & $166.533 \pm 2.616$ & $169.109 \pm 2.992$ \\
Glutamate (ng/g) & $189.686 \pm 1.731$ & $118.114 \pm 2.19$ \\
5-HT (ng/g) & $1.215 \pm 0.34$ & $1.399 \pm 0.62$ \\
5-HIAA (ng/g) & $0.148 \pm 0.01$ & $0.138 \pm 0.028$ \\
Acetylcholine (ng/g) & $6.37 \pm 0.455$ & $6.088 \pm 1.61$ \\
\hline
\end{tabular}

${ }^{* *} p<0.01$ compared with the control group.

Germany) was used to capture images of the IF, ROS, and JC-1 staining. Images were processed using AlphaView SA, Image-Pro Plus, GraphPad Prism, and Photoshop without using touch-up tools, such as cloning and healing tools.

2.16. Statistical Analysis. We used SPSS version 19.0 to analyse the data in this study. The differences in the ADC and FA values among the three groups were analysed using one-way ANOVA followed by Fisher's LSD multiple comparison tests. Additionally, Student's $t$ test was performed to compare the data between the TaClo and control groups. All tests were two-tailed. Statistical significance was defined as $p<0.05$. The data are presented as means \pm standard deviations (SD) of at least three independent experiments.

\section{Results}

3.1. Rats Exhibited Gradual Symptoms of Bradykinesia after TaClo Injection. In the TaClo group, the distance travelled in the open field test showed an evident decreasing trend over time. The ranges of the tracks travelled by the rats in the TaClo group narrowed towards a corner compared to those travelled by the rats in the control group (Figure 1(a)). In addition, the rats in the TaClo group travelled a significantly shorter distance than did the rats in the control group on the 21 st and 28th days (Figure 1(b)). The motor activity decreased by approximately $78.1 \%$ (95\% CI, $71.3 \%$ to $82.5 \%)$ on the 28th day after TaClo treatment. In contrast, rats in the control group did not show any significant difference in the distance travelled at different time points $(p>0.05)$. Based on the video recording, the frequency of spontaneous rotary motion increased on the 28th day after TaClo treatment (Supplementary File 1). According to the results of the behavioural test, the rat PD model was successfully established on the 28th day after TaClo treatment. Therefore, we chose this time point for further study.

3.2. TaClo Induced Demyelination in the Nigrostriatal System. On the 28th day, DTI was performed to examine the nigrostriatal system in the tested rats. In the TaClo group, images of the general outline of the NP showed obvious destruction in the right nigrostriatal cluster, which was not observed in the sham and control groups (Figures 2(a) and 2(b)). Furthermore, the ADC values for the NP were significantly increased in the TaClo-treated rats. No significant changes in the ADC values were detected in the sham and control groups. The FA values for the NP did not differ among the three groups (Figures 2(c) and 2(d)). Based on these DTI results, TaClo impaired the integrity of the nigrostriatal system. Furthermore, the LFB staining revealed demyelination of dopaminergic neurons in the TaClo group consistent with the DTI results (Figure 2(e)).

\subsection{TaClo Decreased the Dopamine Level and Induced the} Loss of Dopaminergic Neurons in the Nigrostriatal System. LC-MS/MS was performed to determine whether the motor dysfunction exhibited by the TaClo-treated rats was accompanied by a decreased striatal DA level. TaClo significantly decreased the DA level in the striatum. The levels of other types of neurotransmitters, including serotonin, glutamate, glutamine, 5-HIAA, and tyramine, did not differ between the two groups (Table 2). The western blot showed significantly decreased $\mathrm{TH}$ levels in the nigrostriatal system after TaClo injection (Figures 3(a) and 3(b)). Meanwhile, the IF images of the full SNc area revealed an apparent loss of TH-positive cells after TaClo treatment (Figure 3(c)). Nissl bodies in the nigrostriatal system were apparently decreased after the TaClo injection (Figures 3(d) and 3(e)). TaClo impaired the function of dopaminergic neurons in the nigrostriatal system in vivo.

3.4. TaClo Induced Mitochondrial Dysfunction by Causing Oxidative Stress. Subsequently, we investigated the mechanism by which TaClo impairs dopaminergic neurons in vivo. By referring to gene expression data obtained from patients with PD in the GEO database (GSE7621-GPL570), which included an analysis of the SNc from patients with $\mathrm{PD}$, the expression of both oxidative stress- and inflammation-related genes was upregulated in the $\mathrm{SN}$ of patients with PD (Figure 4(a)). TaClo also impairs the function of mitochondrial complex I. Therefore, we postulated that the negative effect of TaClo on dopaminergic neurons was associated with oxidative stress. The ATP measurements showed decreased ATP levels after the TaClo treatment (Figure 4(b)). The results of FCM analysis revealed a significantly higher mean fluorescence intensity (MFI) of ROS in the TH-positive cells from the TaClo group than in the control group (Figures 4(c) and 4(d)). These results indicated that TaClo caused oxidative stress in the nigrostriatal system. TaClo impaired the normal function of the mitochondria in dopaminergic neurons. JC-1 staining revealed a decrease in the mitochondrial membrane potential, which is known to occur at the early stage of cell apoptosis (Figures 4(e) and 4(f)).

3.5. TaClo Induced the Innate Immune Response and Apoptosis in the Nigrostriatal System. Images of IHC staining showed increased Iba-1 expression after the TaClo treatment, which is a sign of activated microglia. Usually, resting or quiescent microglia exhibit a branched shape with many synapses. Here, numerous resting microglia transformed to activated microglia characterized by a rounded morphology and dark staining which is known as an amoeboid or phagocytic form after the TaClo treatment (Figure 5(a)). Oxidative stress is known to promote the release of many proinflammatory 


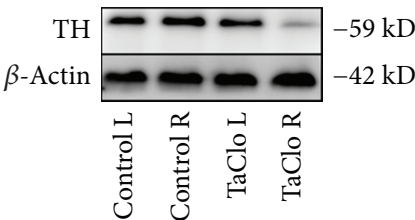

(a)

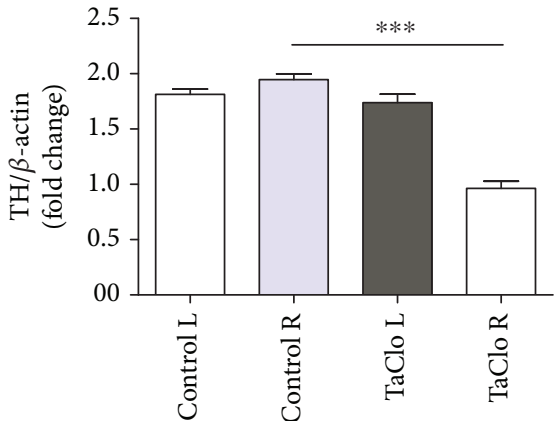

(b)

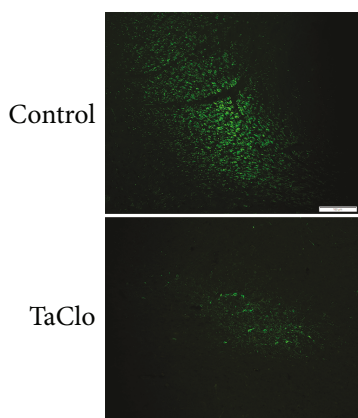

$\mathrm{TH}$

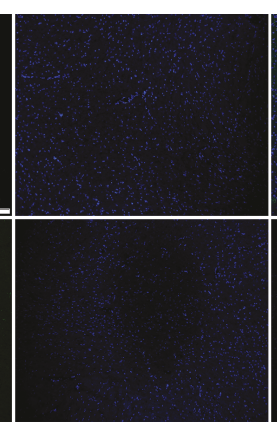

DAPI

(c)

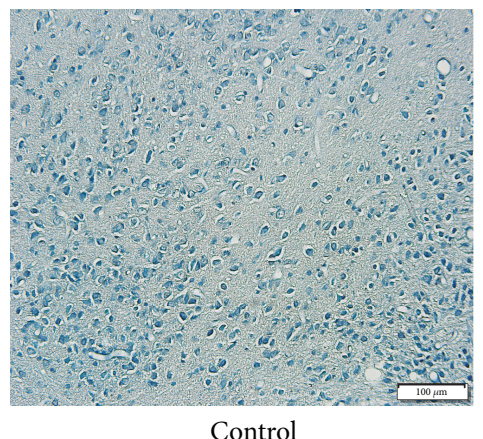

Control

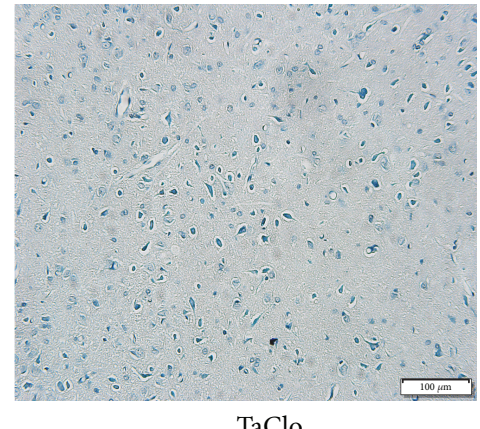

TaClo

(d)

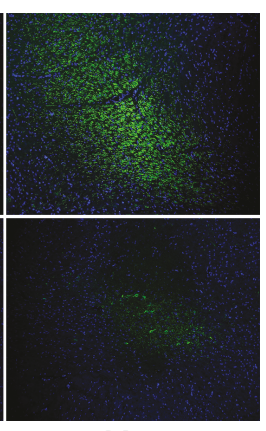

Merge 

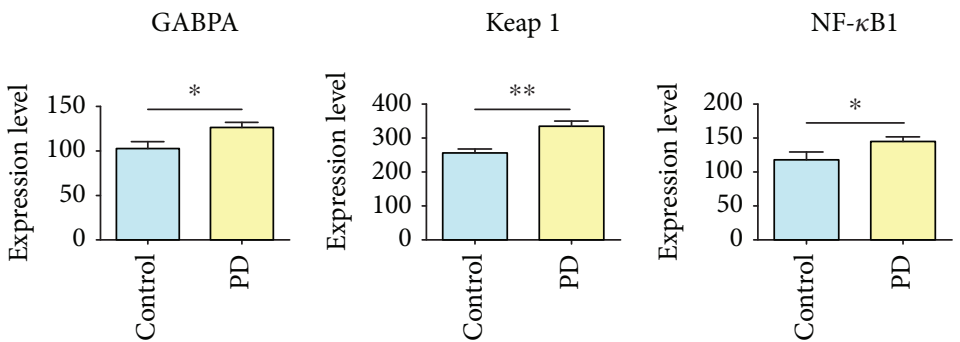

il-6
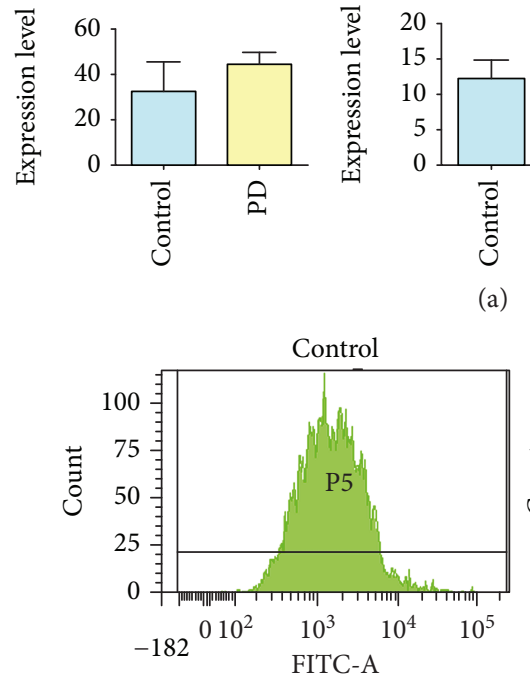

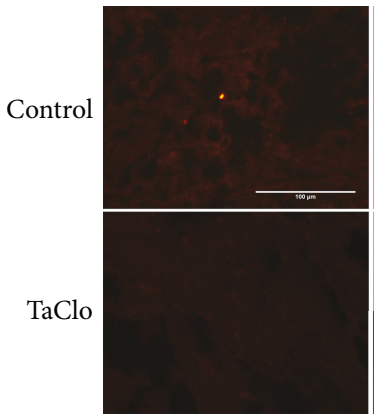

Aggregate

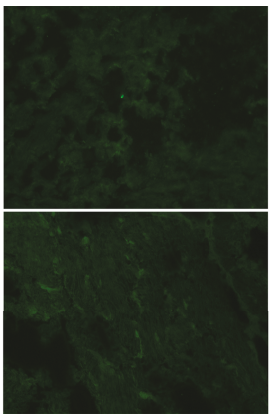

Monomer

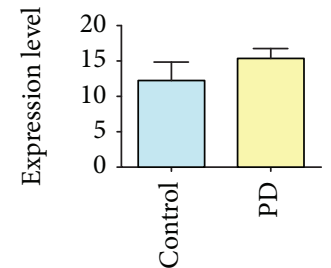

(a)

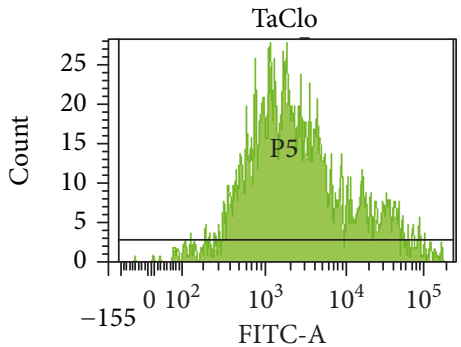

(c)

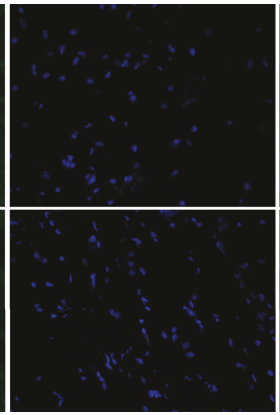

DAPI
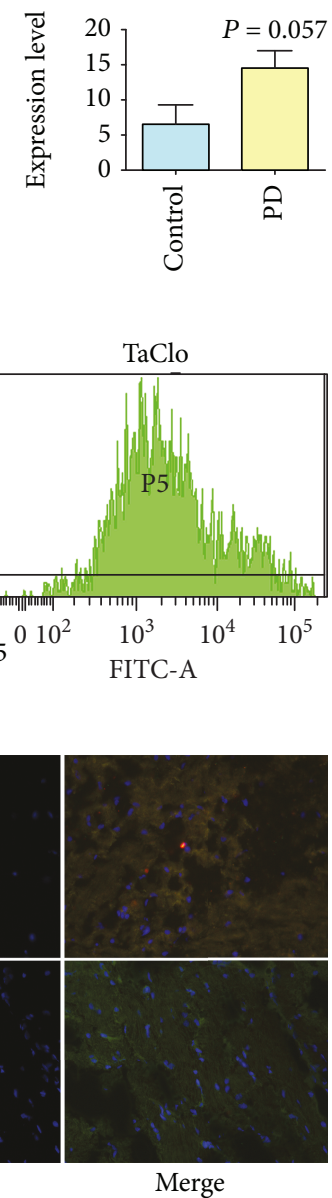

(e)

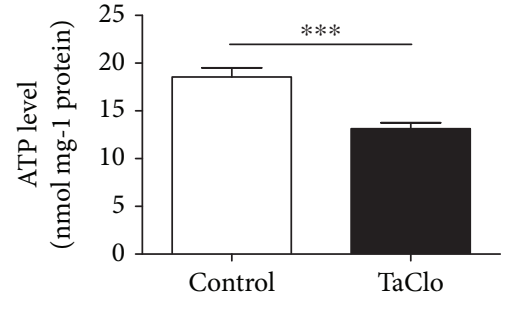

(b)

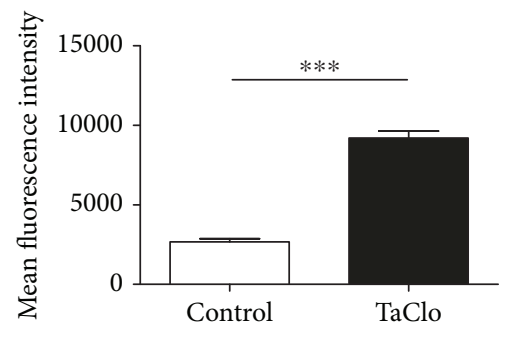

(d)

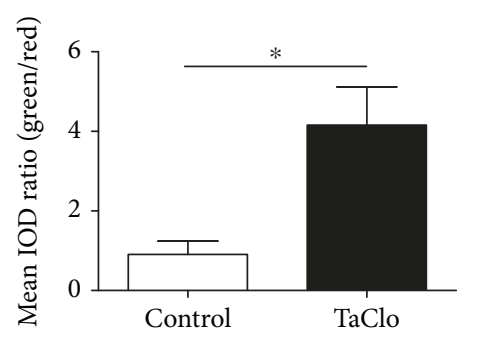

(f)

FIGURE 4: TaClo caused mitochondrial oxidative stress in the nigrostriatal system. (a) The expression of representative genes related to oxidative stress and inflammation from the GEO database (GSE7621-GPL570) was analysed. (b) ATP production was reduced after TaClo treatment. ${ }^{* *} p<0.01$ compared to the control group. (c and d) FCM revealed an apparent increase in the MFI of ROS in the TH-positive cells from the TaClo-treated group compared to the control group. (e and f) Results of JC-1 staining in the two groups. The ratio of green and red fluorescence was increased after the TaClo treatment. ${ }^{*} p<0.05$ compared to the control group. Scale bar $100 \mu \mathrm{m}$.

normal ATP production. According to previous research, the underlying mechanism by which TaClo impaired mitochondrial function is due to the highly lipophilic nature of TCE and TaClo. These compounds easily perturb the lipid bilayers of the celluar and mitochondrial membrane [17]. When TaClo accumulates in the CNS, it is taken up by dopaminergic neurons and is transported across the mitochondrial membrane. Then, it will impair mitochondrial complex I activity and block the electron transport chain, which induces mitochondrial dysfunction, including abnormal ATP production, a decrease in the mitochondrial membrane potential, and oxidative stress. A substantial increase in ROS production is involved in triggering the immune and inflammatory responses [13, 18, 19]. Microglia are cells of mesodermal origin in the brain that are activated under pathological conditions including mitochondrial dysfunction and oxidative stress [20]. When overactivated, microglia uncontrollably release large amounts of ROS and proinflammatory cytokines including IL- 6 , TNF- $\alpha$, Cox- 2 , and iNOS which could exacerbate the loss of dopaminergic 


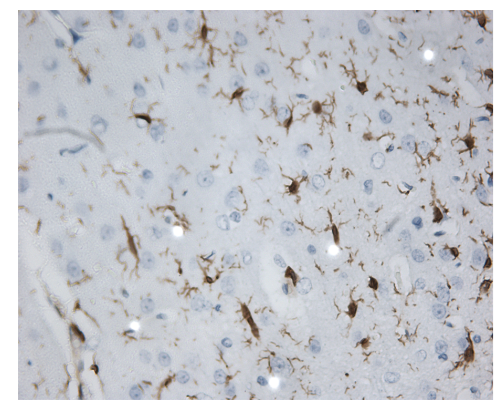

Control

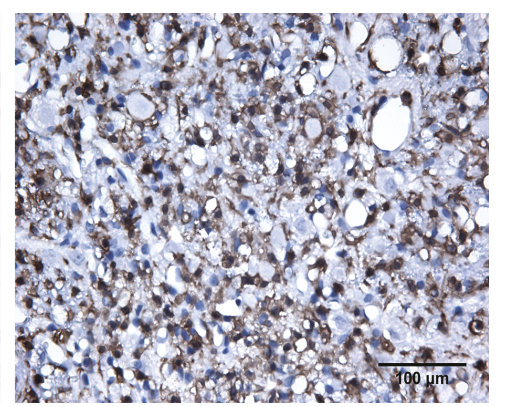

TaClo

(a)

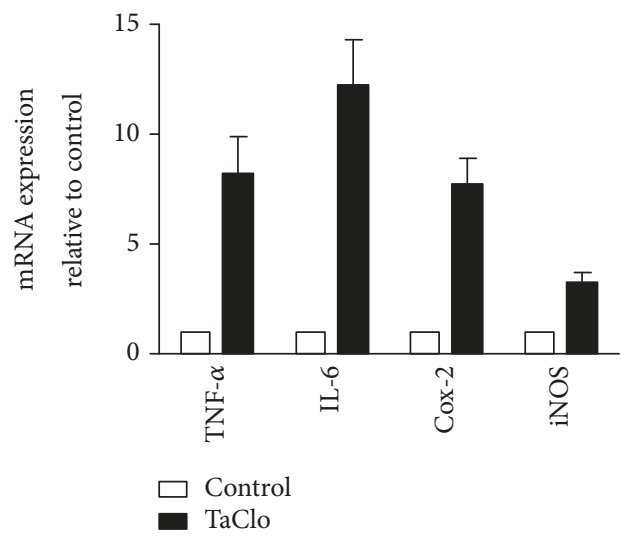

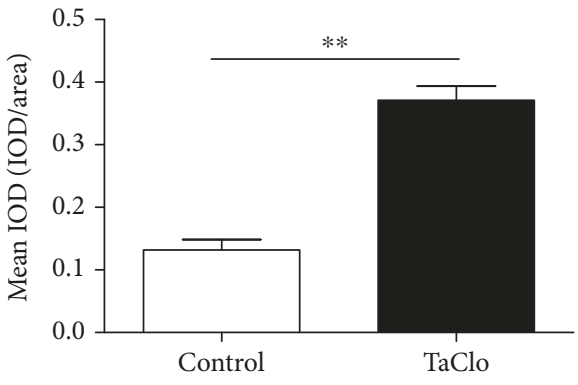

(b)

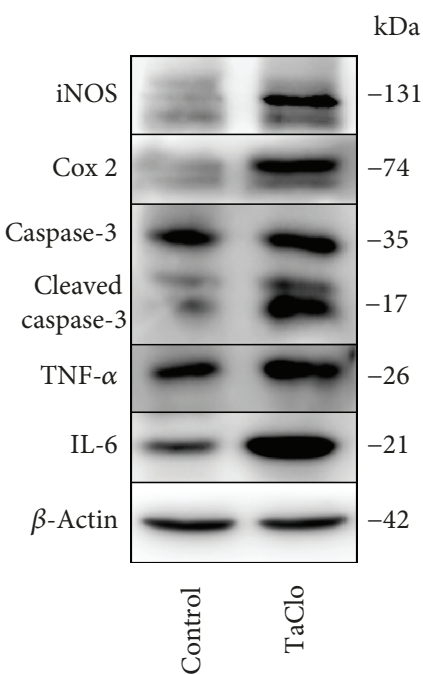

(d)

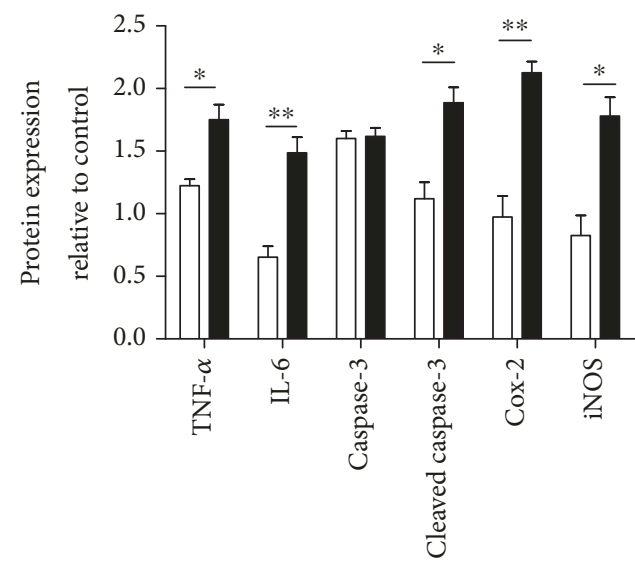

$\square$ Control

(e)

FIgURE 5: The TaClo injection triggered inflammation in the nigrostriatal system. (a) Images of IHC staining showing significantly increased Iba-1 expression in the nigrostriatal system after the TaClo injection $(400 \mathrm{x}) .{ }^{* *} p<0.01$ compared to the control group. (b) RT-PCR results increased levels of the TNF- $\alpha$, IL-6, Cox-2, and iNOS transcripts after TaClo treatment. (c and d) Cropped western blots displaying the levels of the TNF- $\alpha$, IL-6, Cox-2, and iNOS proteins in the nigrostriatal system. ${ }^{*} p<0.05$ and ${ }^{* *} p<0.01$ compared to the control group. Scale bar $100 \mu \mathrm{m}$. 


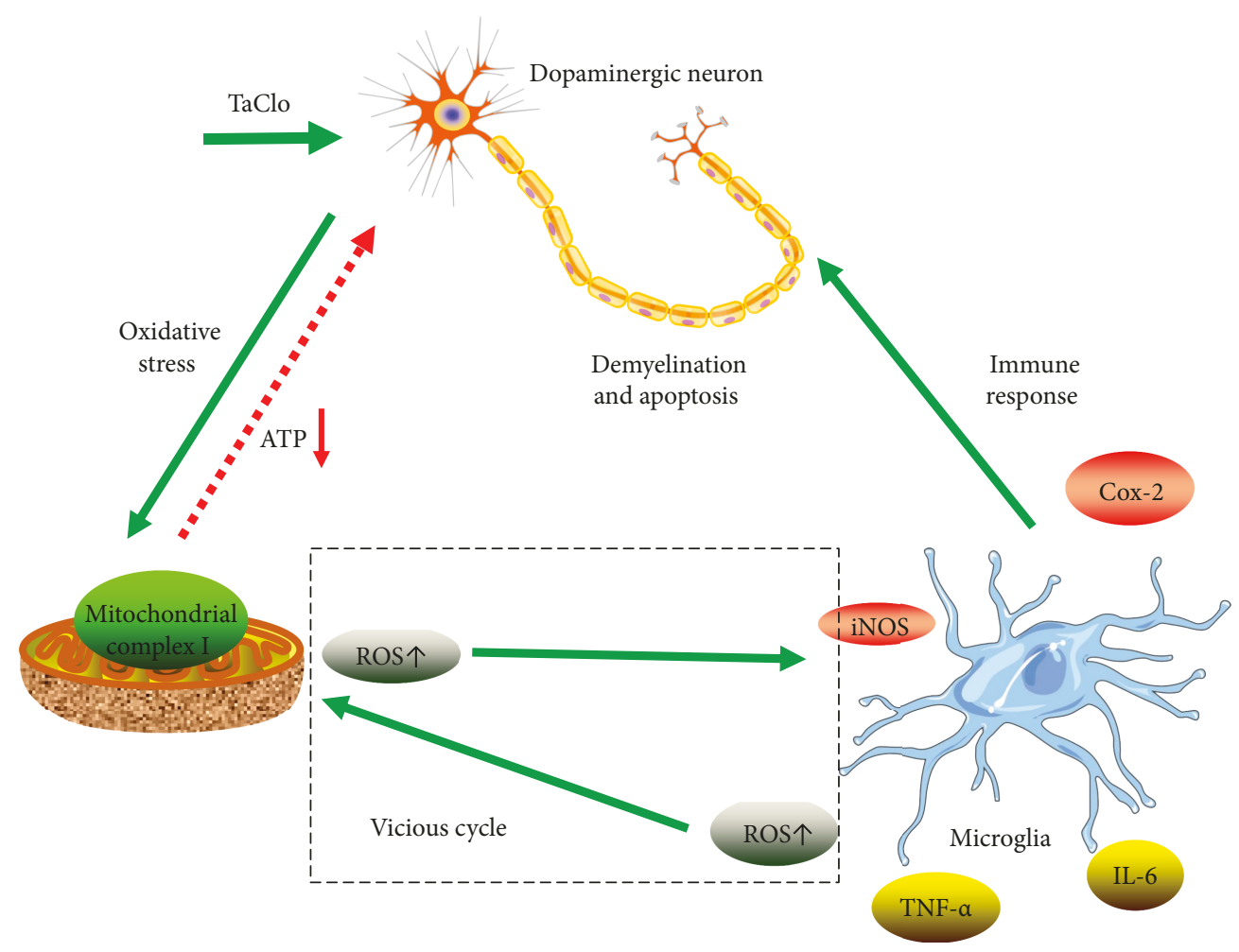

Figure 6: TaClo negatively affected dopaminergic neurons. A schematic diagram showing that TaClo induces the demyelination and apoptosis of dopaminergic neurons by causing mitochondrial dysfunction and triggering the innate immune response in the nigrostriatal system.

neurons [21]. The enzyme iNOS induces the synthesis of the nitric oxide radical, which aggravates oxidative stress and mitochondrial dysfunction. The mitochondrial oxidative stress caused by TaClo activates the innate immune response by activating microglia. Furthermore, the ROS and proinflammatory cytokines released by microglia can exacerbate the mitochondrial dysfunction. As shown in Figure 6, we propose that a positive feedback mechanism exists between mitochondrial oxidative stress and the innate inflammatory response during the process of $\mathrm{TaClo}$ induced $\mathrm{PD}$. This type of loop between oxidative stress and the immune response has been supported by numerous previous articles. Lamb and Goldstein described the interaction between inflammation and oxidative stress as the "oxidative-inflammatory cascade (OIC)" which can lead to many diseases. The OIC is regulated by mediators of the immune and metabolic systems and maintained through a positive feedback loop [22]. According to Voloboueva et al., the mitochondria are both a source of ROS and a critical target of ROS-mediated damage. Mitochondrial ROS production and mitochondrial metabolic disorders are involved in the activation of macrophages called microglia in the CNS [23]. Without any intervention, this vicious cycle can cause serious neural damage since the brain has a lower capacity for cellular regeneration than other organs.

To the best of our knowledge, this study is among the first to identify destruction in the nigrostriatal fibre structure in a neurotoxic rat $\mathrm{PD}$ model based on a voxel-based analysis of DTI. ADC values, but not FA values, for the NP were significantly increased after the TaClo treatment. Our findings are consistent with those reported by Hikishima et al. using a marmoset model treated with MPTP [24]. The increased ADC value indicated a significant increase in the radial diffusivity of water molecules within the nigrostriatal fibres, suggesting that demyelination occurs [25-28]. The LFB staining confirmed that demyelination occurred in the TaClo-treated group, but not in the control group consistent with the DTI results. Based on previous research, mitochondrial dysfunction negatively affects the development of oligodendrocytes and even induces apoptosis in oligodendrocytes, which can further damage the myelin sheath [29]. Therefore, the oxidative stress in mitochondria and innate inflammation induced by TaClo are related to the demyelination of dopaminergic neurons in the nigrostriatal system.

Our study also has some limitations. The present TaClo-based PD model did not show the formation of Lewy bodies which is a pathological hallmark of PD. Based on a previous study, the aggregation of $\alpha$-synuclein and the formation of Lewy bodies are chronic processes [30]. Our rat PD model is an acute model. The early stage of $\mathrm{PD}$ is not accompanied by Lewy pathology [31]. Therefore, a chronic TaClo-based model is needed in subsequent studies to better simulate the natural course of PD 
and evaluate the chronic effect of TaClo on the progression and pathological changes in PD.

Regarding human exposure, TaClo-mediated neurotoxic processes extend over years. Kochen et al. reported an association between the onset of PD in three chronic trichloroethylene-exposed individuals and the presence of TaClo in the ng range [32]. Recently, TaClo has been reported to induce epigenetic carcinogenesis [33]. Therefore, TCE exposure is a public risk. Studies of the toxicity of TaClo are very valuable.

\section{Conclusions}

Our study is among the first to investigate the neurotoxic effects of TaClo, such as causing aggressive $\mathrm{PD}$ in vivo, from the perspective of mitochondrial dysfunction, microglial activation, and the activation of the cascade of inflammatory reactions in the nigrostriatal system. These findings will likely assist with the identification of promising preventative and therapeutic approaches for the treatment of PD.

\section{Data Availability}

The data used to support the findings of this study are available from the corresponding author upon request.

\section{Conflicts of Interest}

The authors have no competing interests to declare.

\section{Acknowledgments}

This work was supported by the National Natural Science Foundation of China (Grant numbers 81100836 and 81771270), the Science and Technology Development Project of Shandong Province, China (Grant number 2014GG B14439); the Natural Science Foundation of Shandong Province (Grant number 2014ZRB14578) and the Promotive Research Fund for Young and Middle-Aged Scientists of Shandong Province (Grant number BS2010YY026).

\section{Supplementary Materials}

Supplementary File 1: video recording of a TaClo-treated rat's spontaneous rotary motions. (A) On the 28th day, a TaClo-treated rat did a number of spontaneous rotary motions which may be caused by weak sense of balance. Meanwhile, the rat stayed in a corner of the testing box. (B) Video recording of a rat in the control group. (Supplementary Materials)

\section{References}

[1] A. Van der Perren, J. Toelen, C. Casteels et al., "Longitudinal follow-up and characterization of a robust rat model for Parkinson's disease based on overexpression of alpha-synuclein with adeno-associated viral vectors," Neurobiology of Aging, vol. 36, no. 3, pp. 1543-1558, 2015.

[2] T. A. Sontag, K. W. Lange, C. Heim, W. Kolasiewicz, O. Tucha, and K. H. Sontag, "Alterations of nocturnal activity in rats following subchronic oral administration of the neurotoxin 1-trichloromethyl-1,2,3,4-tetrahydro- $\beta$-carboline," Journal of Neural Transmission, vol. 116, no. 10, pp. 1267-1271, 2009.

[3] G. Bringmann and A. Hille, "Endogenous alkaloids in man, VII: 1-trichloromethyl-1,2,3,4-tetrahydro- $\beta$-carboline - a potential chloral-derived indol alkaloid in man," Archiv der Pharmazie, vol. 323, no. 9, pp. 567-569, 1990.

[4] G. Bringmann, M. Munchbach, D. Feineis et al., "Endogenous alkaloids in man. XXXVIII. "chiral" and "achiral" determination of the neurotoxin TaClo (1-trichloromethyl-1,2,3,4-tetrahydro- $\beta$-carboline) from blood and urine samples by high-performance liquid chromatography-electrospray ionization tandem mass spectrometry," Journal of Chromatography B, Analytical Technologies in the Biomedical and Life Sciences, vol. 767, no. 2, pp. 321-332, 2002.

[5] G. Bringmann, D. Feineis, M. Munchbach et al., "Toxicity and metabolism of the chloral-derived mammalian alkaloid 1-trichloromethyl-1,2,3,4-tetrahydro- $\beta$-carboline (TaClo) in PC12 cells," Zeitschrift für Naturforschung. Section C, vol. 61, no. 7-8, pp. 601-610, 2006.

[6] B. Janetzky, R. God, G. Bringmann, and H. Reichmann, "1-Trichloromethyl-1,2,3,4-tetrahydro-beta-carboline, a new inhibitor of complex I," Journal of Neural Transmission. Supplementum, vol. 46, pp. 265-273, 1995.

[7] G. Bringmann, R. God, D. Feineis, B. Janetzky, and H. Reichmann, "TaClo as a neurotoxiclead: improved synthesis, stereochemical analysis, and inhibition of the mitochondrial respiratory chain," Journal of Neural Transmission. Supplementum, vol. 46, pp. 245-254, 1995.

[8] D. Huang, J. Xu, J. Wang et al., "Dynamic changes in the nigrostriatal pathway in the MPTP mouse model of Parkinson's disease," Parkinson's Disease, vol. 2017, Article ID 9349487, 7 pages, 2017.

[9] J. Segura-Aguilar, "Aminochrome as preclinical model for Parkinson's disease," Oncotarget, vol. 8, no. 28, pp. 45036-45037, 2017.

[10] M. Liu, E. J. Shin, D. K. Dang et al., "Trichloroethylene and Parkinson's disease: risk assessment," Molecular Neurobiology, vol. 55, no. 7, pp. 6201-6214, 2018.

[11] G. B. Waypa, K. A. Smith, and P. T. Schumacker, "O ${ }_{2}$ sensing, mitochondria and ROS signaling: the fog is lifting," Molecular Aspects of Medicine, vol. 47-48, pp. 76-89, 2016.

[12] L. Liu, K. Zhang, H. Sandoval et al., "Glial lipid droplets and ROS induced by mitochondrial defects promote neurodegeneration," Cell, vol. 160, no. 1-2, pp. 177-190, 2015.

[13] S. Amiri, A. Haj-Mirzaian, M. Momeny et al., "Streptozotocin induced oxidative stress, innate immune system responses and behavioral abnormalities in male mice," Neuroscience, vol. 340, pp. 373-383, 2017.

[14] C. Grote, H. W. Clement, W. Wesemann et al., "Biochemical lesions of the nigrostriatal system by TaClo (1-trichloromethyl-1,2,3,4-tetrahydro-beta-carboline) and derivatives," Journal of Neural Transmission. Supplementum, vol. 46, pp. 275-281, 1995.

[15] G. Bringmann, M. Munchbach, D. Feineis, K. Faulhaber, and H.Ihmels, "Studies on single-strand scissions to cell-freeplasmid DNA by the dopaminergic neurotoxin 'TaClo' (1-trichloromethyl-1,2,3,4-tetrahydro- $\beta$-carboline)," Neuroscience Letters, vol. 304, no. 1-2, pp. 41-44, 2001.

[16] R. S. Akundi, A. Macho, E. Munoz et al., "1-Trichloromethyl-1,2,3,4-tetrahydro- $\beta$-carboline-induced apoptosis in 
the human neuroblastoma cell line SK-N-SH," Journal of Neurochemistry, vol. 91, no. 2, pp. 263-273, 2004.

[17] A. S. Bale, S. Barone Jr, C. S. Scott, and G. S. Cooper, "A review of potential neurotoxic mechanisms among three chlorinated organic solvents," Toxicology and Applied Pharmacology, vol. 255, no. 1, pp. 113-126, 2011.

[18] C. Y. Tseng, J. S. Wang, and M. W. Chao, "Causation by Diesel Exhaust Particles of Endothelial Dysfunctions in Cytotoxicity, Pro-inflammation, Permeability, and Apoptosis Induced by ROS Generation," Cardiovascular Toxicology, vol. 17, pp. 384-392, 2017.

[19] P. Gurung, J. R. Lukens, and T. D. Kanneganti, "Mitochondria: diversity in the regulation of the NLRP 3 inflammasome," Trends in Molecular Medicine, vol. 21, no. 3, pp. 193-201, 2015.

[20] J. L. Marin-Teva, M. A. Cuadros, D. Martin-Oliva, and J. Navascues, "Microglia and neuronal cell death," Neuron Glia Biology, vol. 7, no. 01, pp. 25-40, 2011.

[21] M. A. Panaro and A. Cianciulli, "Current opinions and perspectives on the role of immune system in the pathogenesis of Parkinson's disease," Current Pharmaceutical Design, vol. 18, no. 2, pp. 200-208, 2012.

[22] R. E. Lamb and B. J. Goldstein, "Modulating an oxidative-inflammatory cascade: potential new treatment strategy for improving glucose metabolism, insulin resistance, and vascular function," International Journal of Clinical Practice, vol. 62, no. 7, pp. 1087-1095, 2008.

[23] L. A. Voloboueva, J. F. Emery, X. Sun, and R. G. Giffard, "Inflammatory response of microglial BV-2 cells includes a glycolytic shift and is modulated by mitochondrial glucose-regulated protein 75/mortalin," FEBS Letters, vol. 587, no. 6, pp. 756-762, 2013.

[24] K. Hikishima, K. Ando, R. Yano et al., "Parkinson disease: diffusion MR imaging to detect nigrostriatal pathway loss in a marmoset model treated with 1-methyl-4-phenyl-1,2,3,6-tetrahydropyridine," Radiology, vol. 275, no. 2, pp. 430-437, 2015.

[25] F. Benedetti and I. Bollettini, "Recent findings on the role of white matter pathology in bipolar disorder," Harvard Review of Psychiatry, vol. 22, no. 6, pp. 338-341, 2014.

[26] S. S. Lu, S. J. Kim, N. Kim, H. S. Kim, C. G. Choi, and Y. M. Lim, "Histogram analysis of apparent diffusion coefficient maps for differentiating primary CNS lymphomas from tumefactive demyelinating lesions," American Journal of Roentgenology, vol. 204, no. 4, pp. 827-834, 2015.

[27] S. K. Song, S. W. Sun, M. J. Ramsbottom, C. Chang, J. Russell, and A. H. Cross, "Dysmyelination revealed through MRI as increased radial (but unchanged axial) diffusion of water," NeuroImage, vol. 17, no. 3, pp. 1429-1436, 2002.

[28] F. Branzoli, E. Ercan, R. Valabregue et al., "Differentiating between axonal damage and demyelination in healthy aging by combining diffusion-tensor imaging and diffusion-weighted spectroscopy in the human corpus callosumat 7T," Neurobiology of Aging, vol. 47, pp. 210-217, 2016.

[29] F. Benedetti, S. Poletti, T. A. Hoogenboezem et al., "Inflammatory cytokines influence measures of white matter integrity in bipolar disorder," Journal of Affective Disorders, vol. 202, pp. 1-9, 2016.

[30] K. A. Conway, J. D. Harper, and P. T. Lansbury, "Accelerated in vitro fibril formation by a mutant $\alpha$-synuclein linked to early-onset Parkinson disease," Nature Medicine, vol. 4, no. 11, pp. 1318-1320, 1998.
[31] I.Ferrer, "Early involvement of the cerebral cortex in Parkinson's disease: convergence of multiple metabolic defects," Progress in Neurobiology, vol. 88, no. 2, pp. 89-103, 2009.

[32] W. Kochen, D. Kohlmuller, P. De Biasi, and R. Ramsay, “The endogeneous formation of highly chlorinated tetrahydro- $\beta$-carbolines as a possiblecausative mechanisminidiopathic Parkinson's disease," Advances in Experimental Medicine and Biology, vol. 527, pp. 253-263, 2003.

[33] R. K. Sharma, E. Candelario-Jalil, D. Feineis, G. Bringmann, B. L. Fiebich, and R. S. Akundi, "1-Trichloromethyl-1,2,3,4-tetrahydro-beta-carboline (TaClo) alters cell cycle progression in human neuroblastoma cell lines," Neurotoxicity Research, vol. 32, no. 4, pp. 649-660, 2017. 


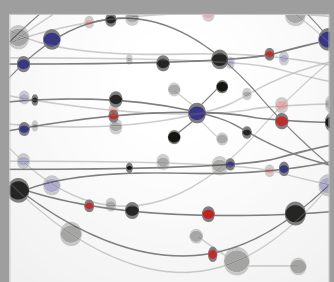

The Scientific World Journal
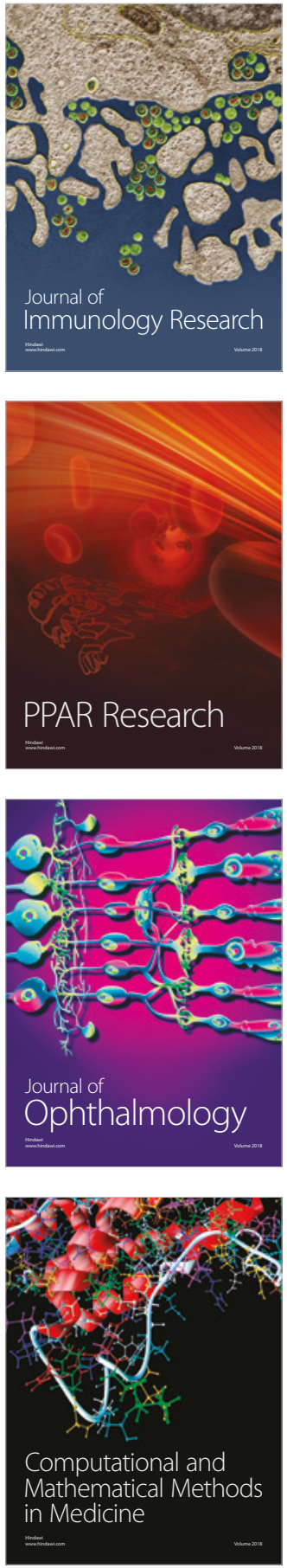

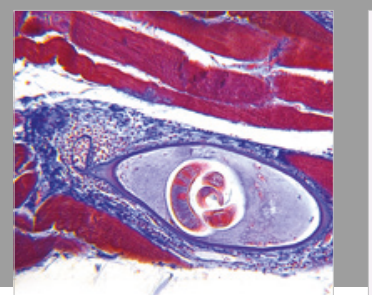

Gastroenterology Research and Practice

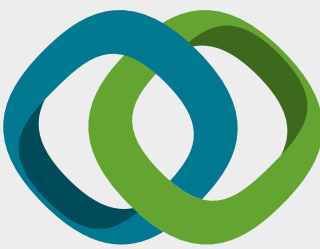

\section{Hindawi}

Submit your manuscripts at

www.hindawi.com
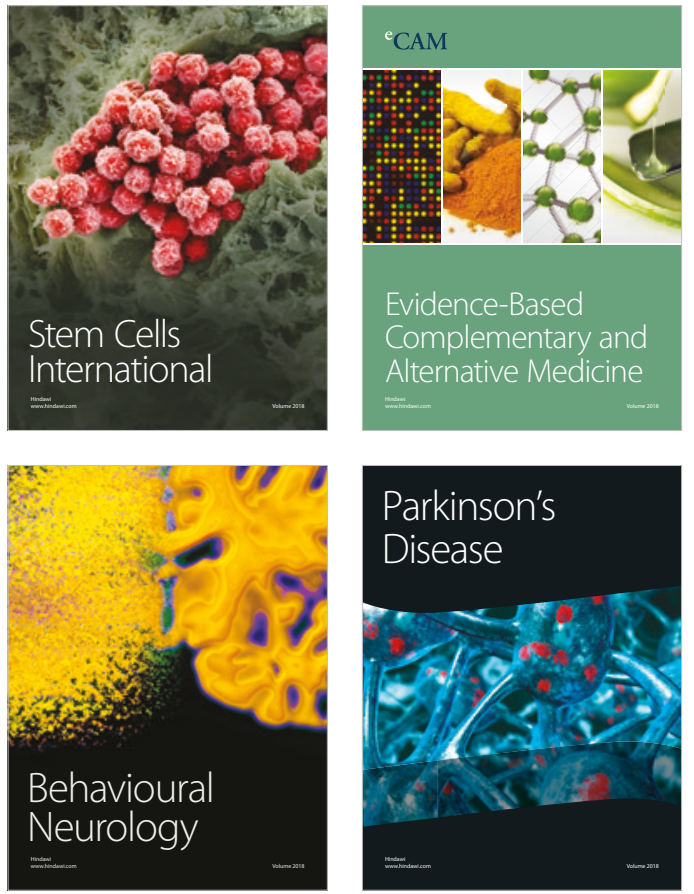

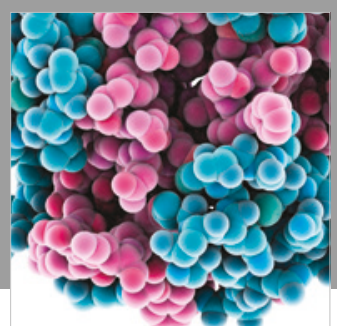

ournal of

Diabetes Research

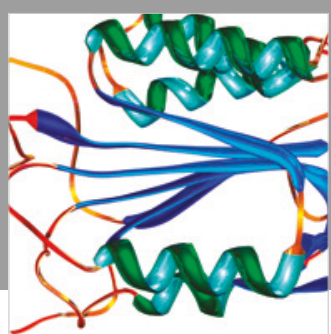

Disease Markers
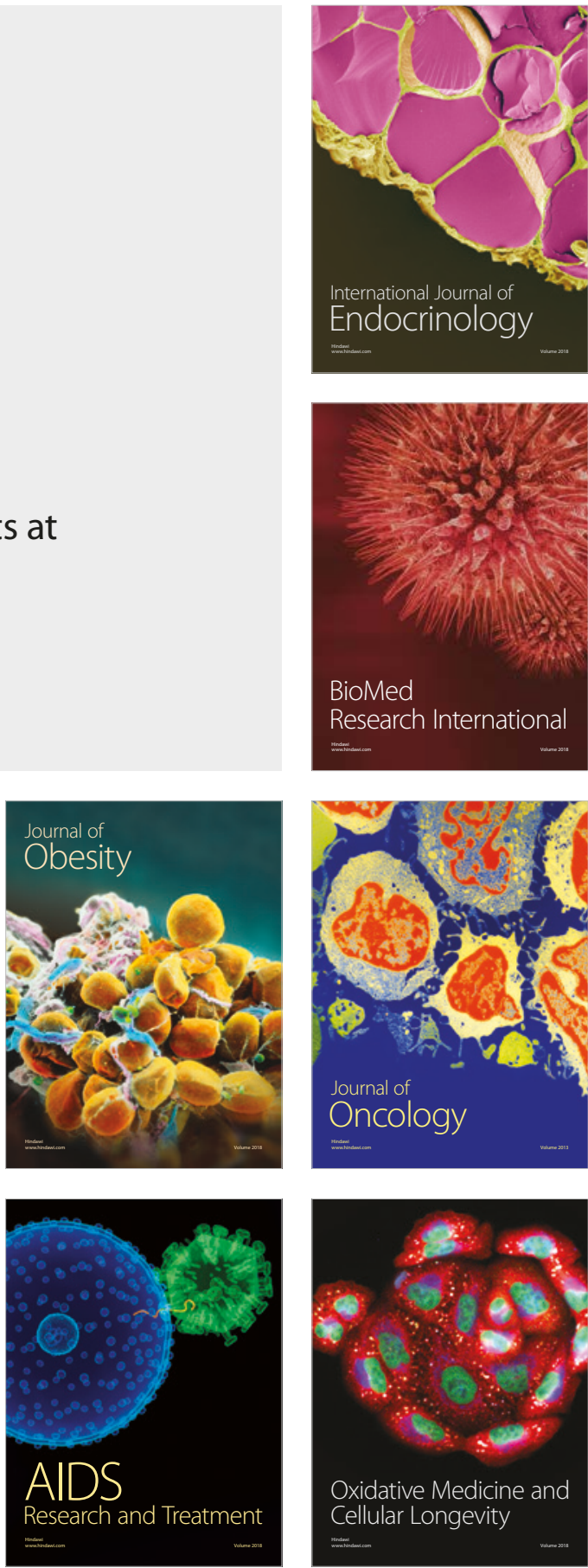